ARTICLE

\title{
Ferroelasticity and domain physics in two-dimensional transition metal dichalcogenide monolayers
}

\author{
Wenbin $\mathrm{Li}^{1} \& \mathrm{Ju} \mathrm{Li}^{2}$
}

\begin{abstract}
Monolayers of transition metal dichalcogenides can exist in several structural polymorphs, including $2 \mathrm{H}, 1 \mathrm{~T}$ and $1 \mathrm{~T}^{\prime}$. The low-symmetry $1 \mathrm{~T}^{\prime}$ phase has three orientation variants, resulting from the three equivalent directions of Peierls distortion in the parental 1T phase. Using first-principles calculations, we predict that mechanical strain can switch the relative thermodynamic stability between the orientation variants of the $1 \mathrm{~T}^{\prime}$ phase. We find that such strain-induced variant switching only requires a few percent elastic strain, which is eminently achievable experimentally with transition metal dichalcogenide monolayers. Calculations indicate that the transformation barrier associated with such variant switching is small ( $<0.2 \mathrm{eV}$ per chemical formula unit), suggesting that strain-induced variant switching can happen under laboratory conditions. Monolayers of transition metal dichalcogenides with $1 \mathrm{~T}^{\prime}$ structure therefore have the potential to be ferroelastic and shape memory materials with interesting domain physics.
\end{abstract}

\footnotetext{
${ }^{1}$ Research Laboratory of Electronics, Massachusetts Institute of Technology, Cambridge, Massachusetts 02139, USA. ${ }^{2}$ Department of Nuclear Science and Engineering and Department of Materials Science and Engineering, Massachusetts Institute of Technology, Cambridge, Massachusetts 02139, USA.

Correspondence and requests for materials should be addressed to J.L. (email: liju@mit.edu).
} 
$\mathrm{T}$ he discovery of two-dimensional (2D) atomic crystals ${ }^{1}$ has fuelled intensive research efforts on this new class of materials, revealing fundamentally new physics and properties $^{2-6}$ that could be essential for next-generation nanoscale devices. Monolayers of group VI transition metal dichalcogenides (TMDs) with chemical formula $\mathrm{MX}_{2}$, where $\mathrm{M}$ is Mo or W and X stands for S, Se or Te, have in particular attracted much recent attention due to their semiconducting, optical and valleytronic properties ${ }^{4,7-9}$. Owning to their atomic thickness, the TMD monolayers have extraordinary mechanical flexibility and strength, capable of sustaining up to $10 \%$ of elastic strain before failure ${ }^{10,11}$, which enables significant dynamical tuning of their properties by strain engineering ${ }^{12}$ and makes them attractive for application in ultrathin flexible electronics ${ }^{13-15}$.

$\mathrm{MX}_{2}$ monolayers can exist in several polytypic structures, including $2 \mathrm{H}, 1 \mathrm{~T}$ and $1 \mathrm{~T}^{\prime 16-18}$. In the semiconducting $2 \mathrm{H}$ phase, the atomic stacking sequence within a single XMX monolayer is Bernal (ABA) and the $\mathrm{M}-\mathrm{X}$ coordination is trigonal prismatic. In contrast, in the $1 \mathrm{~T}$ phase, the XMX stacking sequence is rhombohedral $(\mathrm{ABC})$, and the $\mathrm{M}$ and $\mathrm{X}$ atoms form octahedral coordination. The 1T phase is metallic, but was found to be unstable to Peierls distortion ${ }^{19,20}$, where two adjacent lines of metal atoms along the highest symmetry directions can dimerize and form parallel chains of $\mathrm{M}$ atoms. This leads to the formation of $1 \mathrm{~T}^{\prime}$ phase $\mathrm{s}^{17,18}$, in which the octahedral coordination between $\mathrm{M}$ and $\mathrm{X}$ atoms becomes distorted, and the symmetry of the crystal structure is reduced. While the thermodynamically stable phase of most group VI $\mathrm{MX}_{2}$ monolayers under ambient conditions is $2 \mathrm{H}$, the ground-state phase of $\mathrm{WTe}_{2}$ has $1 \mathrm{~T}^{\prime}$ structure ${ }^{16,21}$. For other $\mathrm{MX}_{2}$ monolayers, the $1 \mathrm{~T}^{\prime}$ phase is usually metastable, but large transition barriers of order $1 \mathrm{eV}$ per formula unit exist between $1 \mathrm{~T}^{\prime}$ and $2 \mathrm{H}$ (ref. 22), suggesting that the $1 \mathrm{~T}^{\prime}$ phase can be stabilized under appropriate thermal or chemical conditions. In particular, the energetic difference between the $2 \mathrm{H}$ and $1 \mathrm{~T}^{\prime}$ phase of $\mathrm{MoTe}_{2}$ is rather small ${ }^{19}$, suggesting that the $1 \mathrm{~T}^{\prime}$ phase can be stabilized relatively easily. Indeed, single crystals and few-layer films of $\mathrm{MoTe}_{2}$ in $1 \mathrm{~T}^{\prime}$ phase have been synthesized on a large scale recently ${ }^{20,23,24}$. It has also been theoretically proposed that the $2 \mathrm{H}$ to $1 \mathrm{~T}^{\prime}$ transition in $\mathrm{MoTe}_{2}$ monolayers can be induced by experimentally accessible tensile strain ${ }^{19}$.

The low-symmetry $1 \mathrm{~T}^{\prime}$ phase of TMD monolayers harbours extraordinary properties that have only started to be revealed, which, for example, includes enhanced catalytic activities ${ }^{25}$, large, non-saturating magnetoresistence ${ }^{21}$ and quantum spin Hall effect ${ }^{22}$.

A ferroelastic material is defined by the existence of two or more equally stable orientation variants, which can be switched from one variant to another without diffusion by the application of external stress ${ }^{26,27}$. A ferroelastic phase usually forms through a structural phase transition (or a hypothetical one) that reduces the symmetry of a prototype phase. The low-symmetry ferroelastic phase possesses several orientation states (domain variants) with different spontaneous $\operatorname{strain}^{28}$, that is, the distortion of the unit cell relative to that in the prototype phase. The difference in spontaneous strain between different variants enables external stress to couple energetically with the strain state of the system and drive orientation switch, analogous to the switching of spontaneous polarization by external electric field in a ferroelectric material. In a ferroelastic crystal, domains of different orientations can coexist and form twin boundaries. On activation by appropriate external stress, those twin boundaries can move in a glissile fashion, resulting in the growth of one orientation state at the expense of another, as well as hysteretic stress-strain response ${ }^{27}$.

In this article, we focus on the possibility of ferroelastic behaviours in $1 \mathrm{~T}^{\prime}-\mathrm{MX}_{2}$ monolayers. A notable feature associated with the $1 \mathrm{~T}^{\prime}$ phase that has hitherto been overlooked is that it has three distinct orientation variants, resulting from the three equivalent directions of structural distortion in the parental $1 \mathrm{~T}$ phase. Our density functional theory (DFT) calculations indicate that ferroelastic switching can occur between the different orientation variants of the $1 \mathrm{~T}^{\prime}$ phase with a few percent of elastic strain, which is experimentally achievable for $\mathrm{MX}_{2}$ monolayers.

\section{Results}

Crystal structures and transformation strains. We use $\mathrm{WTe}_{2}$ monolayers as a representative of $1 \mathrm{~T}^{\prime}-\mathrm{MX}_{2}$ to illustrate the possibility of $2 \mathrm{D}$ ferroelasticity. Figure 1 shows the atomistic structures of $1 \mathrm{~T}-\mathrm{WTe}_{2}$ and $1 \mathrm{~T}^{\prime}-\mathrm{WTe} \mathrm{e}_{2}$ monolayers. In the $1 \mathrm{~T}$ phase, the $\mathrm{W}$ atoms arrange in $2 \mathrm{D}$ triangular lattice, which is sandwiched between two Te atomic layers. The $2 \mathrm{D}$ primitive cell of the $1 \mathrm{~T}$ phase is a $120^{\circ}$ rhombus with side length $t_{0}$. Due to Fermi surface nesting induced Peierls distortion ${ }^{20}$, adjacent parallel lines of $\mathrm{W}$ atoms along the high-symmetry [100], [010] or $[\overline{1} 10]$ directions in the $1 \mathrm{~T}$ structure can spontaneously dimerize and result in the formation of $1 \mathrm{~T}^{\prime}$ phase, with distorted octahedral coordination. The $2 \mathrm{D}$ primitive cell of the $1 \mathrm{~T}^{\prime}$ phase is a rectangle with dimensions $a \times b$, which corresponds to the $1 \times \sqrt{3}$ supercell of the $1 \mathrm{~T}$ phase. Because of the $P \overline{3} m 2$ space group symmetry of the $1 \mathrm{~T}$ phase, there are three symmetry-equivalent directions of structural distortion in the $1 \mathrm{~T}$ phase. These directions are labelled on Fig. 2a as direction 1, 2 and 3 on the 2D triangular lattice formed by $\mathrm{W}$ atoms. The atomistic structures of the three orientation variants formed by structural distortion in the $1 \mathrm{~T}$ phase along the three directions are shown in Fig. 2b-d. Hereafter, we refer to the three orientation states as the $\mathrm{O} 1, \mathrm{O} 2$ and $\mathrm{O} 3$ variant, respectively.

The spontaneous transformation strains associated with the $1 \mathrm{~T}$ to $1 \mathrm{~T}^{\prime}$ transformation can be compared between the three variants based on the $2 \times 2 \sqrt{3}$ supercell of the prototype $1 \mathrm{~T}$ phase. All the three orientation variants of the $1 \mathrm{~T}^{\prime}$ phase, namely, O1, $\mathrm{O} 2$ and $\mathrm{O} 3$, can be derived through the Peierls distortion of this supercell and the atoms within the supercell along the corresponding orientation direction. Namely, the $2 \times 2 \sqrt{3}$

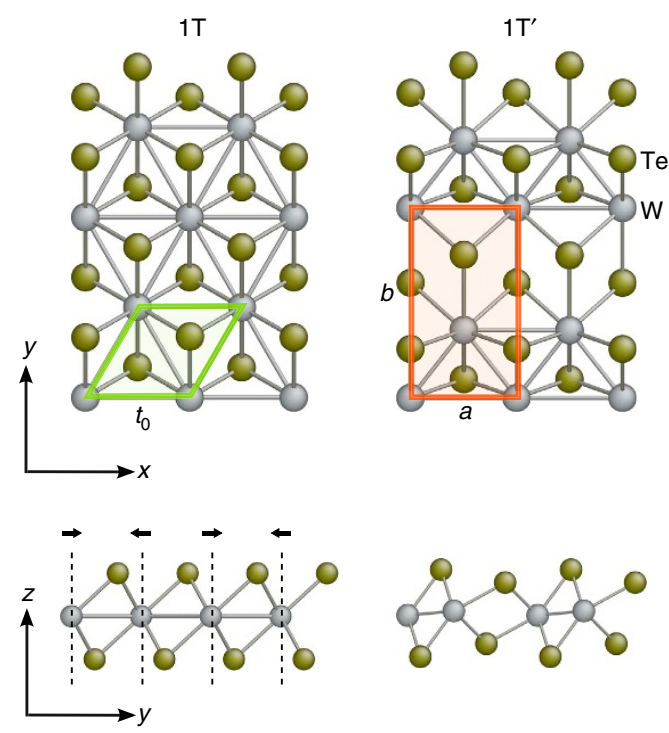

Figure 1 | Atomistic structure of of $1 \mathrm{~T}-\mathrm{WTe} \mathrm{T}_{2}$ and $\mathbf{1 T}^{\prime}-\mathrm{WTe}_{2}$ monolayers. The $2 \mathrm{D}$ primitive cells of $1 \mathrm{~T}$ and $1 \mathrm{~T}^{\prime}$ are highlighted in green and red, respectively. The primitive cell of $1 T^{\prime}$ corresponds to the $1 \times \sqrt{3}$ supercell of $1 \mathrm{~T}$. The $1 \mathrm{~T}^{\prime}$ phase can be derived via the structural distortion of the $1 \mathrm{~T}$ phase, which is schematically illustrated in the side views. These features are generic to all other group $\mathrm{VI} M \mathrm{XX}_{2}$ monolayers. 

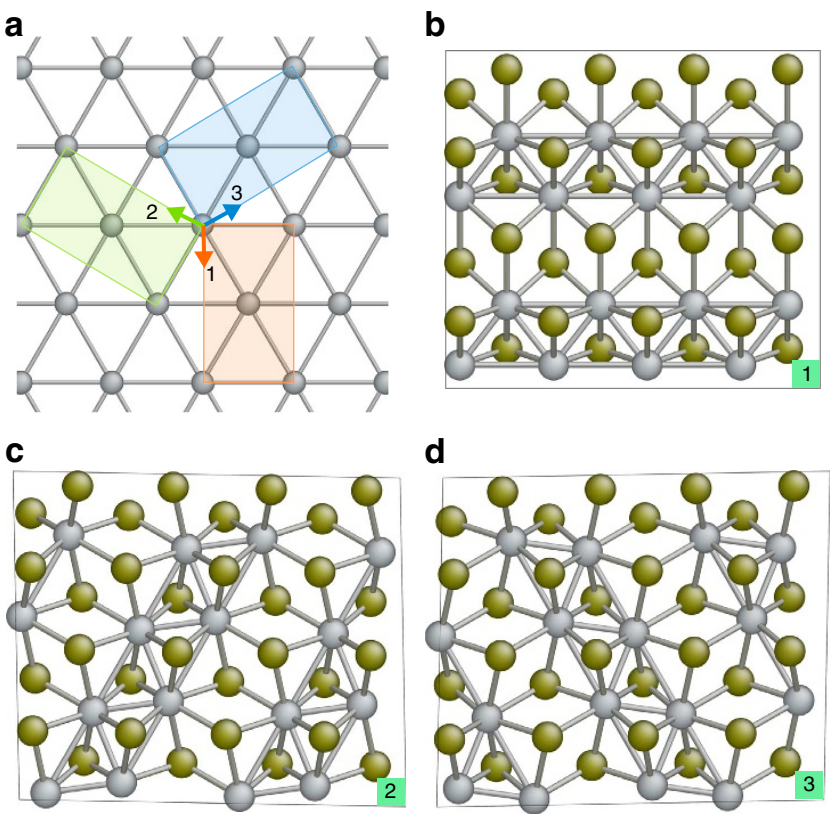

Figure $\mathbf{2}$ | Three orientation variants of $\mathbf{1} \mathbf{T}^{\prime}-\mathbf{M X} \mathbf{2}$ monolayers. (a) The three symmetry-equivalent directions of structural distortion in the $1 \mathrm{~T}$ structure are indicated by arrows and numerical labels on the triangular lattice formed by $\mathrm{M}$ atoms. The corresponding primitive cells of the $1 T^{\prime}$ phase after structural distortion are represented as shaded rectangles. (bd) Relaxed atomistic structure of the $1 \mathrm{~T}^{\prime}$ phase after structural distortion along the three different directions, which are referred to as orientation variants $\mathrm{O} 1, \mathrm{O} 2$ and $\mathrm{O} 3$, respectively.

supercell of $1 \mathrm{~T}$ can transform to become the supercells of all three variants of the $1 \mathrm{~T}^{\prime}$ phase. In Cartesian coordinates, the $2 \mathrm{D}$ basis vectors $\mathbf{h}_{1}$ and $\mathbf{h}_{2}$ of the $1 \mathrm{~T}$ supercell can be written as $\mathbf{h}_{1}=2 t_{0} \hat{\mathbf{x}}$, $\mathbf{h}_{2}=2 \sqrt{3} t_{0} \hat{\mathbf{y}}$, where $\hat{\mathbf{x}}$ and $\hat{\mathbf{y}}$ are the unit vectors along the $x$ and $y$ directions labelled on Fig. 1. A supercell matrix $\mathbf{H}_{0}=\left\{\mathbf{h}_{1}, \mathbf{h}_{2}\right\}$ can be constructed, where $\mathbf{h}_{1}$ and $\mathbf{h}_{2}$ are treated as column vectors, that is,

$$
\mathbf{H}_{0}=\left(\begin{array}{cc}
2 t_{0} & 0 \\
0 & 2 \sqrt{3} t_{0}
\end{array}\right) .
$$

After transforming to the $1 \mathrm{~T}^{\prime}$ phase, the distorted supercell matrix corresponding to the $\mathrm{O} 1, \mathrm{O} 2$ and $\mathrm{O} 3$ variants will be denoted by $\mathbf{H}_{1}, \mathbf{H}_{2}$ and $\mathbf{H}_{3}$, respectively. These new supercell matrices can be related to the original supercell matrix by transformation matrices $\mathbf{J}_{i}$, which map the undistorted supercell to the distorted supercells. Namely, $\mathbf{H}_{i}=\mathbf{J}_{i} \mathbf{H}_{0}$, where the subscript $i$ stands for the $i$-th orientation variant. The transformation strain matrices $\boldsymbol{\eta}_{i}$ associated with different variants can then be calculated from $\mathbf{J}_{i}$ based on the definition of Green-Lagrange strain tensor:

$$
\eta_{i}=\frac{1}{2}\left(\mathbf{J}_{i}^{\mathrm{T}} \mathbf{J}_{i}-\mathbf{I}\right)=\frac{1}{2}\left[\left(\mathbf{H}_{0}^{-1}\right)^{\mathrm{T}} \mathbf{H}_{i}^{\mathrm{T}} \mathbf{H}_{i} \mathbf{H}_{0}^{-1}-\mathbf{I}\right] .
$$

Here, the superscripts -1 and $\mathrm{T}$ denote matrix inversion and transposition, respectively. $\mathbf{I}$ is a $2 \times 2$ identity matrix. The $2 \mathrm{D}$ transformation strain tensor $\boldsymbol{\eta}_{i}$ has the following symmetric form:

$$
\eta=\left(\begin{array}{ll}
\varepsilon_{x x} & \varepsilon_{x y} \\
\varepsilon_{x y} & \varepsilon_{y y}
\end{array}\right),
$$

where $\varepsilon_{x x}$ and $\varepsilon_{y y}$ are the tensile/compressive strain along $x$ or $y$ direction, and $\varepsilon_{x y}$ is the shear strain component.

We have employed DFT calculations to obtain the equilibrium supercell vectors and the relaxed atomic coordinates of the O1, $\mathrm{O} 2$ and $\mathrm{O} 3$ variants, resulting from the distortion of $2 \times 2 \sqrt{3}$ supercell in the $1 \mathrm{~T}$ prototype phase. The supercell matrices $\mathbf{H}_{i}$ for different $\mathrm{MX}_{2}$ monolayers are tabulated in Supplementary Table 1. From the supercell matrices, the spontaneous transformation strain tensors $\boldsymbol{\eta}_{i}$ can be evaluated, which are listed in Supplementary Table 2. For $\mathrm{WTe}_{2}$ monolayers, the transformation strain matrices form $1 \mathrm{~T}$ to $1 \mathrm{~T}^{\prime}$ are

$$
\begin{aligned}
\eta_{1} & =\left(\begin{array}{cc}
-0.005 & 0.0 \\
0.0 & 0.039
\end{array}\right), \\
\eta_{2} & =\left(\begin{array}{cc}
0.029 & -0.019 \\
-0.019 & 0.006
\end{array}\right), \\
\eta_{3} & =\left(\begin{array}{ll}
0.029 & 0.019 \\
0.019 & 0.007
\end{array}\right) .
\end{aligned}
$$

The difference in transformation strain between the three variants of $1 \mathrm{~T}^{\prime}$ suggests that one may switch the relative thermodynamic stability between different variants by applying suitable external mechanical stress. Since the equilibrium structure of $\mathrm{WTe}_{2}$ is $1 \mathrm{~T}^{\prime}$, it is informative to directly compare the distorted supercell of the three variants by computing the relative supercell strain associated with the transformation from one variant to another. This can be carried out again using the supercells of the three variants derived from the common $2 \times 2 \sqrt{3}$ supercell in the prototype $1 \mathrm{~T}$ phase. The reference configuration for computing the supercell strain is now chosen to be the O1 variant of $1 \mathrm{~T}^{\prime}$ phase, and we use $\varepsilon_{i}^{j}$ to denote the transformation strain tensor from variant $i$ to $j$. Calculations based on the same definition of strain tensor as in equation (2) give the transformation strain associated with $\mathrm{O} 1-\mathrm{O} 2$ and $\mathrm{O} 1-\mathrm{O} 3$ switching to be

$$
\begin{gathered}
\varepsilon_{1}^{2}=\left(\begin{array}{cc}
0.034 & -0.019 \\
-0.019 & -0.030
\end{array}\right), \\
\varepsilon_{1}^{3}=\left(\begin{array}{cc}
0.033 & 0.019 \\
0.019 & -0.030
\end{array}\right) .
\end{gathered}
$$

It then follows that, starting with the $\mathrm{O} 1$ variant of $1 \mathrm{~T}^{\prime}$ in a strainfree state, after imposing an external strain of magnitude $\varepsilon_{1}^{2}$ on the monolayer, the system would be in a thermodynamically more favourable state by transforming to the $\mathrm{O} 2$ variant, since both $\mathrm{O} 1$ and $\mathrm{O} 2$ belong to the same $1 \mathrm{~T}^{\prime}$ structure, but $\mathrm{O} 1$ will have higher strain energy than $\mathrm{O} 2$. The same argument applies to any other two variants. Hence, the relative energetic stability between the different orientation variants of $1 \mathrm{~T}^{\prime}$ phase can be controlled by external stress or strain.

Variant energetics under biaxial and shear strain. To study in detail the relative thermodynamic stability of different variants when external mechanical deformation is imposed on a $1 \mathrm{~T}^{\prime}-\mathrm{MX}_{2}$ monolayer, we have used DFT to calculate the potential energy surfaces of the three variants of $1 \mathrm{~T}^{\prime}$ as a function of $2 \mathrm{D}$ supercell dimensions. We first investigate the possibility of mechanically switching the $\mathrm{O} 1$ variant to $\mathrm{O} 2$ or $\mathrm{O} 3$ variant by applying biaxial strain to the system, again using $\mathrm{WTe}_{2}$ monolayer as an example. The strain-free $2 \times 2$ supercell of the $\mathrm{O} 1$ variant, derived from the distortion of the aforementioned $2 \times 2 \sqrt{3}$ supercell in the parental $1 \mathrm{~T}$ phase, is chosen to be the reference system. The $2 \times 2$ supercell of the $\mathrm{O} 1$ variant has dimensions $2 a \times 2 b$ within the $x-y$ plane of 2D monolayer. We adjust the dimensions of the supercell along $x$ and $y$ directions independently, with the values of $a$ and $b$ range from -10 to $10 \%$ of engineering strain at an equal step of $2 \%$. At each pair of $(a, b)$, the atomic coordinates within the supercell are relaxed. We also compute the energies of $\mathrm{O} 2$ and $\mathrm{O} 3$ variants when their supercell dimensions are fixed to be the same as $\mathrm{O} 1$. The energies $U$ of all three variants are computed on a 


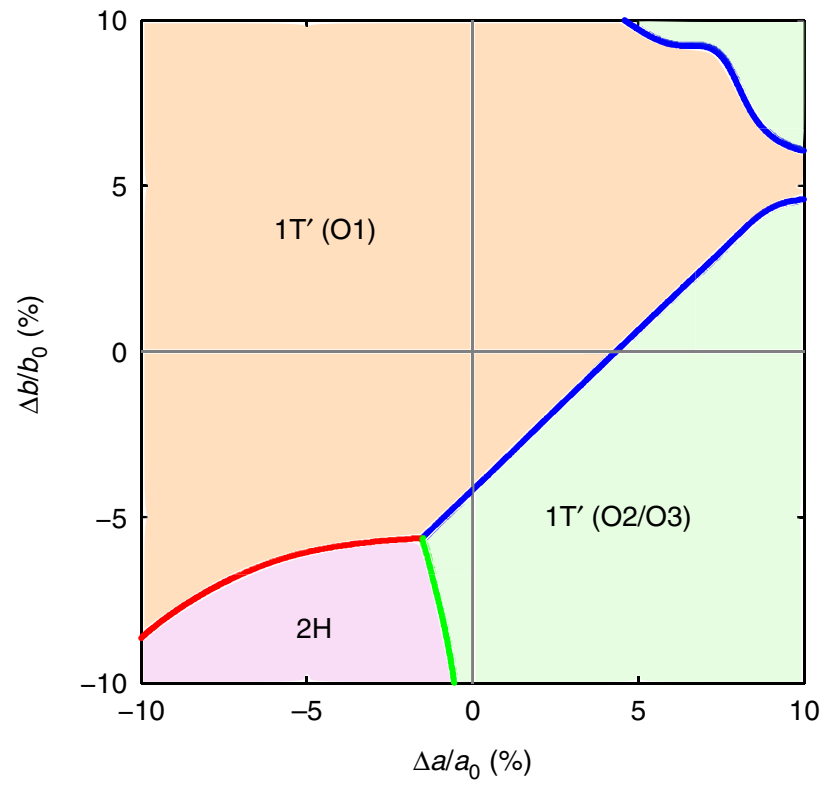

Figure 3 | Intersection contours of the energy surfaces between the different orientation variants of $\mathbf{1 T}^{\prime}-\mathbf{M o T e}_{\mathbf{2}}$ and between $\mathbf{2 H}$ and $\mathbf{1 T}^{\prime}$. The lattice constants $a$ and $b$, corresponding to the dimensions of the rectangular primitive cell of the $\mathrm{O} 1$ variant in the $1 \mathrm{~T}^{\prime}$ phase, are represented as percent engineering strain with respect to the equilibrium lattice constants $a_{0}$ and $b_{0}$. The regions of lower-energy phase/variant are labelled and shaded in different colours.

$11 \times 11$ grid in the $(a, b)$ space, giving a total number of 121 data points distributed evenly around the equilibrium lattice constants of the O1 variant. Smooth potential energy surfaces are then constructed by approximating the intermediate values of $U(a, b)$ using 2D spline interpolation, which allows us to directly compare the relative energetic stability of the $\mathrm{O} 1, \mathrm{O} 2$ and $\mathrm{O} 3$ variants in the full $(a, b)$ space. In addition, the $U(a, b)$ for the $2 \mathrm{H}$ phase is computed for comparison, as a previous study indicates that strain-induced phase transformation between the $2 \mathrm{H}$ and $1 \mathrm{~T}^{\prime}$ phases can happen in $\mathrm{MX}_{2}$ monolayers ${ }^{19}$.

After obtaining the potential energy surfaces for all the three variants of $1 \mathrm{~T}^{\prime}$ as well as the $2 \mathrm{H}$ phase, the lowest-energy variants/phases in the $(a, b)$ space are determined. The result is shown in Fig. 3, where we label the lowest-energy variant/phase in each region of phase space and plot the intersection boundaries between two neighbouring variants/phases. An important feature of Fig. 3 is that the potential energy surfaces of $\mathrm{O} 1$ and $\mathrm{O} 2 / \mathrm{O} 3$ variants intersect at a few percent of biaxial supercell strain, which is experimentally achievable in $\mathrm{MX}_{2}$ monolayers ${ }^{10,11}$. The $\mathrm{O} 2$ and $\mathrm{O} 3$ variants are grouped together in Fig. 3 because their potential energies in the $(a, b)$ space are essentially the same. This can be rationalized by the fact that the supercells of both variants can be derived from the distortion of the $2 \times 2 \sqrt{3}$ supercell in the $1 \mathrm{~T}$ phase, and their distortion directions are related by mirror symmetry along the $y$ direction in the $1 \mathrm{~T}$ phase, as can be seen from Fig. 2. Since biaxial strain does not break the mirror symmetry of $1 \mathrm{~T}$ phase along the $y$ axis, the $\mathrm{O} 2$ and $\mathrm{O} 3$ variants are still mirror images of each other and have the same energy. We however expect that shear strain, which breaks the mirror symmetry, can distinguish the energies of all the three variants of $1 \mathrm{~T}^{\prime}$. Indeed, Fig. 4 shows that, when shear strain $\varepsilon_{x y}$ of magnitude $>3.5 \%$ is imposed on the $\mathrm{O} 1$ variant, $\mathrm{O} 3$ becomes the lowest-energy variant within the strained supercell. If the sign of $\varepsilon_{x y}$ is reversed, then the $\mathrm{O} 2$ variant has lower energy than both $\mathrm{O} 1$ and $\mathrm{O} 3$.

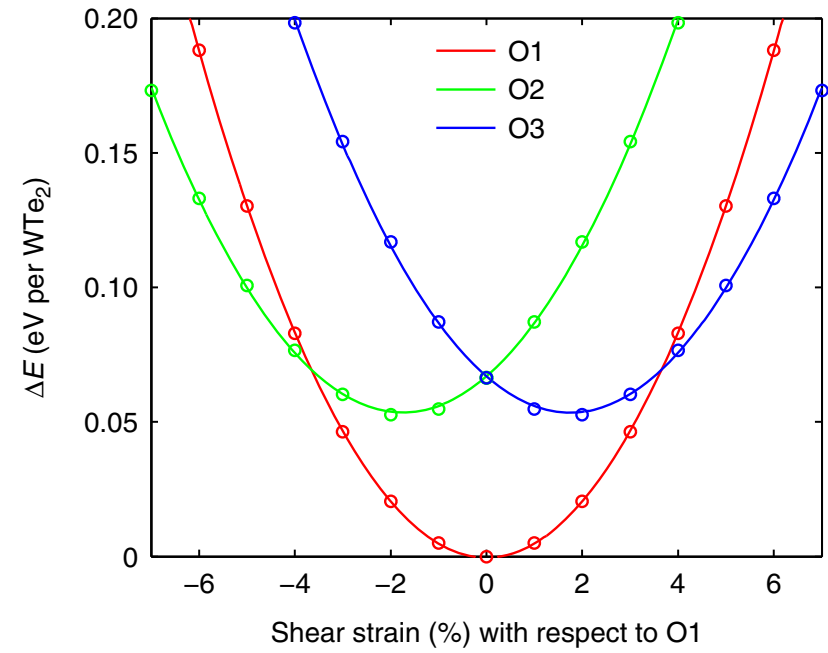

Figure 4 | Potential energies of the three variants of $\mathbf{1 T}^{\prime}-\mathrm{WTe}_{2}$ monolayers as a function of shear strain with respect to the equilibrium supercell of the $\mathbf{0 1}$ variant. The $\mathrm{O} 2$ or $\mathrm{O} 3$ variant becomes energetically more favourable when negative- or positive-shear strain of a few percent is imposed on the 01 variant.

Figure 3 indicates that the $2 \mathrm{H}$ phase of $\mathrm{WTe}_{2}$ monolayer only takes a small region in the $(a, b)$ space as the lowest-energy phase. This result is different from the study by Duerloo et al. ${ }^{19}$ of strain-induced phase transformation between the $2 \mathrm{H}$ and $1 \mathrm{~T}^{\prime}$ phases of $\mathrm{MX}_{2}$ monolayers, as the authors did not take into account the existence of orientation variant degrees of freedom in the $1 \mathrm{~T}^{\prime}$ phase.

Figure 3 also shows that the fastest route to switching the energetic order between $\mathrm{O} 1$ and $\mathrm{O} 2 / \mathrm{O} 3$ in the $(a, b)$ space is by applying tensile strain along the $a$ axis of the $\mathrm{O} 1$ variant, which is the direction of dimerized metal-atom chains, while simultaneously applying compressive strain along the $b$ axis. It is however known that $2 \mathrm{D} \mathrm{MX}_{2}$ monolayers usually cannot sustain large compressive strain due to compression-induced buckling response and formation of ripplocations ${ }^{29}$. On the contrary, experiments have demonstrated that $2 \mathrm{H}-\mathrm{MX}_{2}$ monolayers can withstand tensile elastic strain as large as $10 \%$ before mechanical failure $e^{10,11}$. Hence, it may be experimentally more convenient to realize variant switching in $1 \mathrm{~T}^{\prime}-\mathrm{WTe}_{2}$ by uniaxially stretching it along the $a$ axis, which is the direction of dimerized tungsten atoms. This axis can be identified by mechanical cleavage or by the anisotropic response to external fields that is expected for the low-symmetry $1 \mathrm{~T}^{\prime}$ structure ${ }^{21}$.

In Supplementary Fig. 1, we have also computed the intersection contours of the potential energy surfaces between the $\mathrm{O} 1$ and $\mathrm{O} 2 / \mathrm{O} 3$ variants for other $1 \mathrm{~T}^{\prime}-\mathrm{MX}_{2}$ monolayers, including $\mathrm{MoS}_{2}, \mathrm{MoSe}_{2}, \mathrm{MoTe}_{2}, \mathrm{WS}_{2}$ and $\mathrm{WSe}_{2}$. The results are very similar to $\mathrm{WTe}_{2}$, indicating that strain-induced switching of thermodynamic stability between different orientation variants is generic to $\mathrm{MX}_{2}$ monolayers with $1 \mathrm{~T}^{\prime}$ structure.

Variant energetics under uniaxial tension. We emphasize that the strain at which the potential energy surfaces of different variants intersects is not the same as the strain at which variant switching becomes thermodynamically favourable. The system can minimize its free energy by choosing a state where different variants (or phases) coexist, akin to the two-phase region in chemical-composition phase diagrams. Under constant temperature and fixed external strain (supercell dimensions), the thermodynamic potential that determines the relative variant/ 


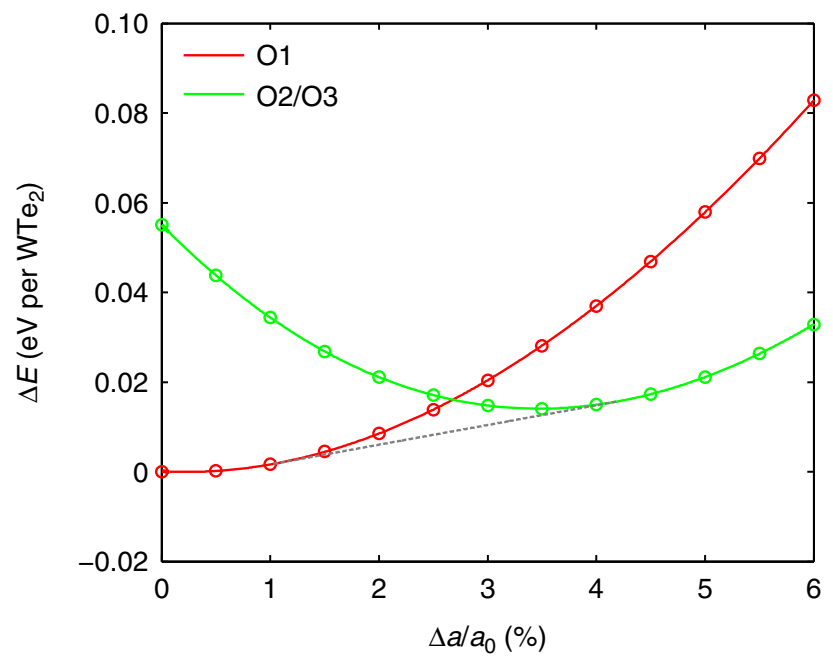

Figure 5 | Potential energy curves of the different variants of $1 \mathbf{1 T}^{\prime}-\mathrm{WTe}_{\mathbf{2}}$ uniaxially strained along the $\boldsymbol{a}$ axis. The dashed line is the common tangent between the two curves. The uniaxial strain is calculated with respect to the equilibrium supercell dimension of the $\mathrm{O} 1$ variant along the $x$ direction.

phase stability is the Helmholtz free energy $F=E-T S$, where $E$ is internal energy that includes both potential energy $U$ and kinetic energy, $T$ is temperature and $S$ is entropy. Because all the three variants $\mathrm{O} 1, \mathrm{O} 2$ and $\mathrm{O} 3$ belong to the same $1 \mathrm{~T}^{\prime}$ structure, and because the entropy of solids (mainly vibrational) is relatively insensitive to small deformation, we can use the potential energy $U$ of different variants, computed by DFT at zero temperature, to compare the free energies of different variants at ambient conditions. In Fig. 5, we plot the potential energy curve of the $\mathrm{O} 1$ variant of $1 \mathrm{~T}^{\prime}-\mathrm{WTe}_{2}$ when it is uniaxially stretched along the $a$ axis. Consistent with typical experimental set-ups for uniaxial deformation, the stress of the supercell along the $b$ axis is relaxed to zero. This corresponds to free boundary, or zero stress $\left(\sigma_{y}=0\right)$ condition along the $b$ axis. In Fig. 5, we also plot the potential energy of the $\mathrm{O} 2 / \mathrm{O} 3$ variant in a rectangular supercell with the same dimension along the $a$ axis and the same boundary condition along the $b$ axis. A common tangent can be constructed between the energy curves of $\mathrm{O} 1$ and $\mathrm{O} 2 / \mathrm{O} 3$, which intersects the two curves at uniaxial strains equal to $1 \%$ and $4 \%$, respectively. Between these two values, the system can lower its energy by existing in a state where both $\mathrm{O} 1$ and $\mathrm{O} 2 / \mathrm{O} 3$ variants coexist. This indicates that the formation of $\mathrm{O} 2 / \mathrm{O} 3$ variants becomes thermodynamically favourable when the uniaxial strain along the $a$ axis of $\mathrm{O} 1$ is as low as $1 \%$.

Kinetic aspects of variant switching. Up to now, we have only considered the thermodynamic aspects of variant switching in the $1 \mathrm{~T}^{\prime}-\mathrm{MX}_{2}$ monolayers. Our results suggest that it becomes thermodynamically favourable for the $\mathrm{O} 1$ variant of $1 \mathrm{~T}^{\prime}-\mathrm{WTe}_{2}$ monolayers to switch to the other two variants when applying uniaxial strain around $1 \%$ along the direction of dimerized tungsten atom chains. However, if the kinetic barrier associated with variant switching is too high, such variant switching may not occur under normal experimental conditions and timescale, and the materials would still not be ferroelastic. We have therefore computed the transition barrier associated with the switching between the $\mathrm{O} 1, \mathrm{O} 2$ and $\mathrm{O} 3$ variants using climbing image nudged elastic band (NEB) method $^{30}$. The result of our calculation for the variant switching between the $\mathrm{O} 1$ and $\mathrm{O} 2$ variants of $\mathrm{WTe}_{2}$ monolayer is shown in Fig. 6. We find the transition barrier of variant switching is only $0.22 \mathrm{eV}$ per formula unit. Very similar results are obtained for orientation switching between other variants, as presented in Supplementary Fig. 2. Note that to facilitate these NEB calculations, we impose supercell strains on the $\mathrm{O} 2$ or $\mathrm{O} 3$ variants such that they have the same supercell dimensions of the $\mathrm{O} 1$ variants. The strain energy results in the slightly higher energy of the $\mathrm{O} 2$ or $\mathrm{O} 3$ variant that would otherwise be energetically degenerate with the $\mathrm{O} 1$ variant. In Supplementary Fig. 3, we have also computed the transition barrier and the pathway between stress-free $\mathrm{O} 1$ and $\mathrm{O} 2$ variants using generalized solid-state NEB method ${ }^{31}$, which allows both the atomic and supercell degrees of freedom to relax along the transition pathway. The results of the generalized solid-state NEB calculation are very close to those obtained using a fixed-supercell approach, with the calculated energy barrier of variant switching equals to $0.19 \mathrm{eV}$ per formula unit. We note that, while the transition pathway illustrated in Fig. 6 may not be the only possible one, if other pathways exist, the transformation barrier of variant switching can only be smaller or equal than the values we have obtained.

In Supplementary Fig. 4, we have also computed the transition barriers for other $\mathrm{MX}_{2}$ monolayers, and the barriers obtained are even lower than $1 \mathrm{~T}^{\prime}-\mathrm{WTe}_{2}$ monolayers. The transition barriers of variant switching are significantly lower than the barriers of phase transition between the $2 \mathrm{H}$ and $1 \mathrm{~T}^{\prime}$ phase ${ }^{19}$, which we computed to be $0.8 \mathrm{eV}$ per formula unit for $1 \mathrm{~T}^{\prime}-\mathrm{WTe}_{2}$ monolayers at the equilibrium lattice constants of the $2 \mathrm{H}$ phase. The much smaller transition barriers associated with the variant switching within the $1 \mathrm{~T}^{\prime}$ phase as compared with the $1 \mathrm{~T}^{\prime}$ to $2 \mathrm{H}$ phase transition has an intuitive geometric explanation. Variant switching between the orientation variants of $1 \mathrm{~T}^{\prime}$ phase only involves the distortion of $\mathrm{M}-\mathrm{X}$ octahedral coordination, while the $1 \mathrm{~T}^{\prime}$ to $2 \mathrm{H}$ phase transition requires the complete change of $\mathrm{M}-\mathrm{X}$ coordination pattern from octahedral to trigonal prismatic.

According to transition-state theory, assuming a characteristic attempt frequency of $10 \mathrm{THz}$, which is the typical frequency of optical phonons in $1 \mathrm{~T}^{\prime}-\mathrm{MX}_{2}$ monolayers ${ }^{22}$, a $0.2 \mathrm{eV}$ barrier is associated with a timescale of around $0.2 \mathrm{~ns}$. Although the actual barrier of forming a critical nucleus of new variant may involve multiple formula units, and other factors such as interfaces and pre-existing defects may also affect the transformation kinetics, the much smaller barrier associated with variant switching within the $1 \mathrm{~T}^{\prime}$ phase as compared with $2 \mathrm{H}$ to $1 \mathrm{~T}^{\prime}$ phase transition ${ }^{19,20}$ suggests that ferroelastic variant switching in $1 \mathrm{~T}^{\prime}-\mathrm{MX}_{2}$ monolayers is very likely to happen under normal laboratory experimental conditions.

Ferroelastic domain boundaries. A direct consequence of straininduced variant switching in $1 \mathrm{~T}^{\prime}-\mathrm{MX}_{2}$ monolayers is the formation of domain boundaries between different orientation variants. Strain-induced ferroelastic switching between the O1, $\mathrm{O} 2$ and $\mathrm{O} 3$ variants can lead to the formation of three possible types of coherent twin boundaries, between $\mathrm{O} 1$ and $\mathrm{O} 2, \mathrm{O} 1$ and $\mathrm{O} 3$, and between $\mathrm{O} 2$ and $\mathrm{O} 3$, which we refer to as $\mathrm{O} 1-\mathrm{O} 2$, O1-O3 and O2-O3, respectively. The DFT-relaxed atomistic structures of the three different types of twinning domain boundaries in $1 \mathrm{~T}^{\prime}-\mathrm{WTe}_{2}$ monolayers under zero external stress are shown in Fig. 7. The three domain boundaries are energetically degenerate, and they are related to each other by $120^{\circ}$ rotational symmetry operation. Unlike their three-dimensional (3D) counterparts, where the domain boundaries are $2 \mathrm{D}$, the boundaries formed between the domains of $2 \mathrm{D} \mathrm{MX}_{2}$ monolayers are quasi-one dimensional (1D) in nature, which may impart them unique properties. We have calculated the domain boundary energies associated with the three types of the $1 \mathrm{D}$ domain boundaries and found they have small formation energies. Our DFT calculations give the domain boundary energies of $\mathrm{MoS}_{2}$, 


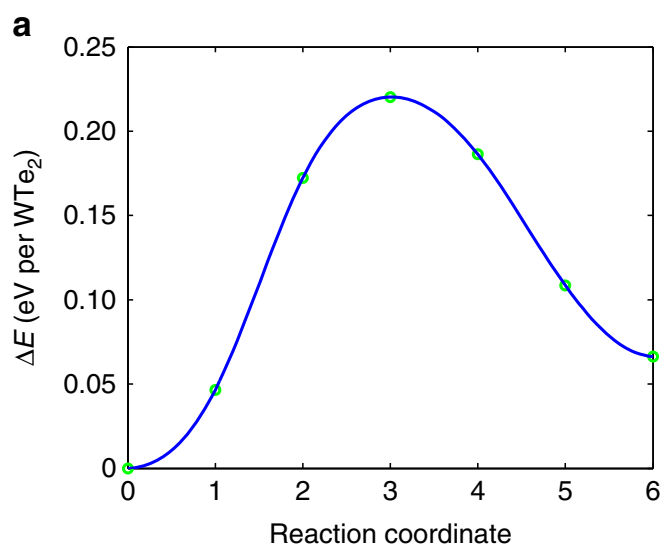

b 1

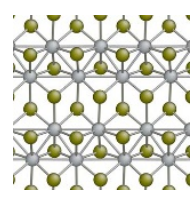

4

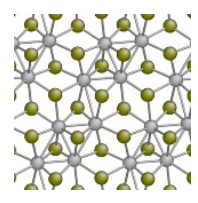

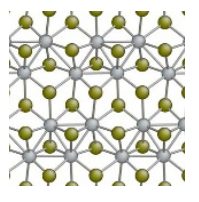

5

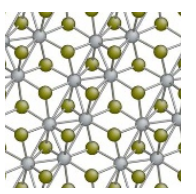

3

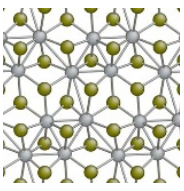

6

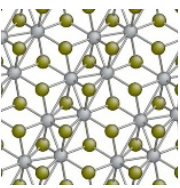

Figure 6 | NEB calculation of transformation barrier and the pathway for orientation switching. The initial configuration of the NEB calculation is the $\mathrm{O} 1$ variant of $1 \mathrm{~T}^{\prime}-\mathrm{WTe} \mathrm{T}_{2}$ monolayers at ground state (zero external stress), while the final state is the strained $\mathrm{O} 2$ variant with the same supercell dimensions as those of the $\mathrm{O} 1$ variant. (a) Change of the system energy per chemical formula unit as a function of reaction coordinate. (b) The corresponding atomistic structure of the system along the reaction coordinate. Four different supercells were used to carry out the NEB calculations: $2 \times 2,2 \times 4,4 \times 2$ and $4 \times 4$ supercell of the $1 T^{\prime}$ phase, which all give identical results.

a

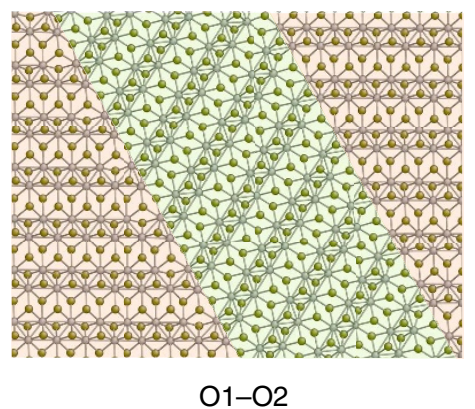

b

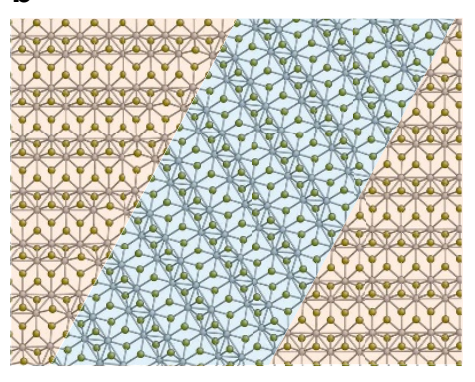

$\mathrm{O} 1-\mathrm{O} 3$
C

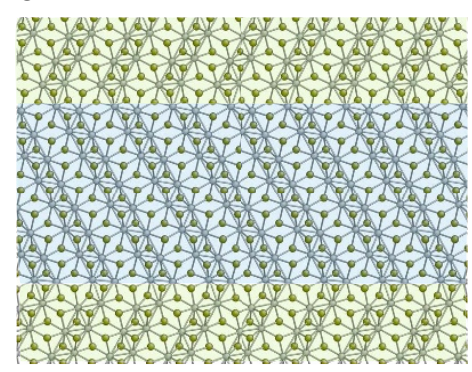

$\mathrm{O} 2-\mathrm{O} 3$

Figure 7 | Ferroelastic domain boundaries between different orientation variants. (a-c) Illustrate the DFT-relaxed atomistic structures of domain boundaries formed between the $\mathrm{O} 1$ and $\mathrm{O} 2, \mathrm{O} 1$ and $\mathrm{O} 3$, and $\mathrm{O} 2$ and $\mathrm{O} 3$ variants of $1 \mathrm{~T}^{\prime}-\mathrm{WTe}_{2}$ monolayers, respectively. To help guide the eyes, domains of different variants are shaded in distinct colours: $\mathrm{O} 1$ variant in orange, $\mathrm{O} 2$ variant in green and $\mathrm{O} 3$ variant in blue.

$\mathrm{MoSe}_{2}, \mathrm{MoTe}_{2}, \mathrm{WS}_{2}, \mathrm{WSe}_{2}$ and $\mathrm{WTe} \mathrm{T}_{2}$ monolayers to be 27, 46, $40,22,51$ and $52 \mathrm{meV} \cdot \AA^{-1}$, respectively. In comparison, the formation energy of another type of $1 \mathrm{D}$ defects in $3 \mathrm{D}$ crystals, dislocations, is in the order of several hundred meV per Angstrom. Such small-domain boundary energies will facilitate the ferroelastic switching between different orientation variants.

\section{Discussion}

The thermodynamic and kinetic analysis above have provided strong evidence that strain-induced ferroelastic switching of orientation variants can occur in $1 \mathrm{~T}^{\prime}-\mathrm{MX}_{2}$ monolayers, with a few percent of local strain. Our calculations indicate that variant switching can most easily happen when stretching the $1 \mathrm{~T}^{\prime}-\mathrm{MX}_{2}$ monolayers along the direction of dimerized metal chains. This prediction, if experimentally realized, will render $1 \mathrm{~T}^{\prime}-\mathrm{MX}_{2}$ monolayers as the first class of $2 \mathrm{D}$ ferroelastic materials ${ }^{32}$. Signatures of such ferroelastic switching in experiments include hysteresis in stress-strain curves ${ }^{27}$, and the existence of a force plateau when the externally applied strain is beyond a critical value that corresponds to the onset of variant coexistence. Direct experimental proof of ferroelastic domain switching may be realized by carrying out in situ transmission electron microscopy experiments of mechanical deformation of $1 \mathrm{~T}^{\prime}-\mathrm{MX}_{2}$ monolayers. As the domains of different variants have different crystallo- graphic orientations, the migration of domain walls during straininduced variant switching can be observed by dark-field transmission electron microscopy, which has been demonstrated for domain imagining in graphene and $\mathrm{MoS}_{2}$ monolayers $^{33,34}$. Selective area electron diffraction could also reveal the formation of twinning domains, as variant switching results in the rotation of the underlying Bravais lattice of the $1 \mathrm{~T}^{\prime}$ structure, which will manifest in selective area electron diffraction as the rotation of diffraction patterns.

Our prediction of ferroelasticity in the TMD monolayers can be readily tested experimentally in $1 \mathrm{~T}^{\prime}-\mathrm{WTe}_{2}$ and $1 \mathrm{~T}^{\prime}-\mathrm{MoTe}_{2}$, for which bulk single crystals have been synthesized on a large scale and exfoliated down to the monolayer or few-layer regime 20,21 . Recently, large-area and high-quality $\mathrm{MoTe}_{2}$ few layers in $1 \mathrm{~T}^{\prime}$ phase have been grown via chemical vapour deposition ${ }^{23,24}$. Local and controlled phase transformation of $\mathrm{MoTe}_{2}$ from the $2 \mathrm{H}$ to $1 \mathrm{~T}^{\prime}$ phase can also be realized using laser ablation ${ }^{35}$. In principle, ferroelastic domain switching can be observed not only in monolayers but also in few-layer samples, since dimerized metal chains within different layers of the $1 \mathrm{~T}^{\prime}$ phase orient along the same direction in naturally grown crystals ${ }^{36}$.

For other group VI MX 2 that include $\mathrm{MoS}_{2}, \mathrm{MoSe}_{2}, \mathrm{WS}_{2}$ and $\mathrm{WSe}_{2}$, as the $2 \mathrm{H}$ phase is energetically more stable than the $1 \mathrm{~T}^{\prime}$ phase under normal conditions, the $1 \mathrm{~T}^{\prime}$ phase can be realized using a phase engineering approach ${ }^{37,38}$. The $1 \mathrm{~T}$ or $1 \mathrm{~T}^{\prime}$ phase of 
these materials are now actively being explored for applications in energy and electronics ${ }^{38}$. Monolayers of $\mathrm{WS}_{2}, \mathrm{MoS}_{2}$ and $\mathrm{MoSe}_{2}$ in $1 \mathrm{~T}^{\prime}$ phase have been obtained via liquid-phase exfoliation of the bulk crystals intercalated by alkaline metals ${ }^{18,25,39}$. The transformation from the $2 \mathrm{H}$ phase to $1 \mathrm{~T}^{\prime}$ phase by alkaline metal intercalation is attributed to charge transfer from the intercalated alkali atoms to the TMDs ${ }^{37}$. We have performed DFT calculations to study the effect of lithium atom adsorption on the relative energetics of $2 \mathrm{H}-\mathrm{MX}_{2}$ and $1 \mathrm{~T}^{\prime}-\mathrm{MX}_{2}$ monolayers, and it is indeed shown that, with increased amount of adsorbed lithium, the $1 \mathrm{~T}^{\prime}$ phase becomes energetically more favourable than the $2 \mathrm{H}$ phase for all the $\mathrm{MX}_{2}$ monolayers, as illustrated in Supplementary Fig. 5. It has also been proposed that the substitutional doping of $\mathrm{MX}_{2}$ with elements having more valence electrons (for example, Re) than the transition metal ions can be another effective way to stabilize the $1 \mathrm{~T}^{\prime}$ phase ${ }^{40}$.

From an application perspective, ferroelastic behaviours have close connection to the shape memory effect, which has been exploited to make actuators in a wide range of industries. In 3D, shape memory alloys (SMAs) is a well known and technologically important class of ferroelastic materials. SMAs can undergo a diffusionless martensitic phase transformation below a critical temperature, from the high-temperature austenite to the lowtemperature martensite phase ${ }^{41}$. The martensite phase of SMAs is ferroelastic: it has several equivalent orientations or variants, that can be switched from one to another by an appropriate uniaxial or shear stress. The martensite phase in SMAs can undergo large inelastic deformation through stress-induced migration of twin boundaries between different variants. On heating the deformed crystal above the martensitic phase transformation temperature, the martensite phase can revert to the austenite phase and recover its original shape before deformation. For the ferroelastic $1 \mathrm{~T}^{\prime}-\mathrm{MX}_{2}$ monolayers, if the $1 \mathrm{~T}^{\prime}$ phase can be reversibly transformed to the $1 \mathrm{~T}$ or $2 \mathrm{H}$ phase under external stimuli (which do not have to be thermal but could be other fields), then $\mathrm{MX}_{2}$ monolayers could be $2 \mathrm{D}$ shape memory materials, with operating principles similar to SMAs. As such, $1 \mathrm{~T}^{\prime}-\mathrm{MX}_{2}$ monolayers can be used to make ultrathin actuators for applications in nanoscale-integrated electromechanical systems.

In closing, we would like to make a few additional comments. First, since the $1 \mathrm{~T}^{\prime}-\mathrm{MX}_{2}$ monolayers were predicted to be quantum spin Hall insulators ${ }^{22}$, topological effect plays an important role in determining their electronic properties. The twinning domain boundaries formed through ferroelastic switching in the $1 \mathrm{~T}^{\prime}$ phase, which are $1 \mathrm{D}$ defects in $2 \mathrm{D}$ quantum materials, may possess exotic physics and provide a rich playground for domain boundary engineering ${ }^{42}$. Second, the possibility of ferroelastic switching in 2D materials may not be limited to group VI $\mathrm{MX}_{2}$ monolayers, considering the rich family of $2 \mathrm{D}$ materials ${ }^{43}$. Our initial studies indicate that several other TMD monolayers, including $\mathrm{ReS}_{2}, \mathrm{NbTe}_{2}$ and $\mathrm{TaTe}_{2}$, which have low-symmetry distorted crystal structures similar to $1 \mathrm{~T}^{\prime 16}$, also possess distinct orientation variants and could be ferroelastic as well. Indeed, experimental evidence of local strain-induced orientational switching in $\mathrm{ReS}_{2}$ and $\mathrm{ReSe}_{2}$ monolayers has recently been reported ${ }^{44}$. Our finding of potential 2D ferroelastic behaviours in monolayer materials could therefore open doors to many exciting discoveries in $2 \mathrm{D}$ materials with low-symmetry distorted crystal structures, which may also include ferroelectric, ferromagnetic and multiferroic behaviours in the future.

\section{Methods}

First-principles calculations. DFT calculations were performed using the Vienna $\mathrm{Ab}$ initio Simulation Package with a plane-wave basis set ${ }^{45,46}$ and the projector- augmented wave ${ }^{47}$ pseudopotentials. Exchange-correlation effects were treated using the generalized gradient approximation ${ }^{48}$ in the Perdew-Burke-Ernzerhof form $^{49}$. The kinetic energy cutoff for wavefunction expansion was fixed to be $350 \mathrm{eV}$. The TMD monolayers were modelled in supercells with a vacuum region in the direction perpendicular to the 2D planes of the monolayers (the $z$ direction). The length of the supercells along the $z$ direction was chosen to be $20 \AA$. Brillouin zone integration employed a Gamma point centred $m \times n \times 1$ Monkhorst-Pack $^{50}$ k-point grid and a Gaussian smearing of $50 \mathrm{meV}$, where the numbers $m$ and $n$ were chosen such that the $\mathbf{k}$-point sampling spacing is $<0.1 \AA^{-1}$ along the supercell reciprocal vectors in the $x-y$ plane. The energy convergence thresholds for electronic and ionic relaxations were $10^{-6}$ and $10^{-5} \mathrm{eV}$, respectively. The maximum residual forces resulted from these convergence criteria are smaller than $5 \times 10^{-3} \mathrm{eV}^{-1}$. We provide the DFT-relaxed atomistic structures of the O1, $\mathrm{O} 2$ and $\mathrm{O} 3$ variants of $1 \mathrm{~T}^{\prime}-\mathrm{MX}_{2}$ monolayers in the POSCAR format of Vienna $\mathrm{Ab}$ initio Simulation Package, as listed in Supplementary Tables 4-9.

\section{References}

1. Novoselov, K. S. et al. Two-dimensional atomic crystals. Proc. Natl Acad. Sci. USA 102, 10451-10453 (2005).

2. Castro Neto, A. H., Guinea, F., Peres, N. M. R., Novoselov, K. S. \& Geim, A. K. The electronic properties of graphene. Rev. Mod. Phys. 81, 109-162 (2009).

3. Lee, C., Wei, X. D., Kysar, J. W. \& Hone, J. Measurement of the elastic properties and intrinsic strength of monolayer graphene. Science 321, 385-388 (2008).

4. Wang, Q. H., Kalantar-Zadeh, K., Kis, A., Coleman, J. N. \& Strano, M. S. Electronics and optoelectronics of two-dimensional transition metal dichalcogenides. Nat. Nanotechnol. 7, 699-712 (2012).

5. Xu, X., Yao, W., Xiao, D. \& Heinz, T. F. Spin and pseudospins in layered transition metal dichalcogenides. Nat. Phys. 10, 343-350 (2014).

6. Xia, F., Wang, H., Xiao, D., Dubey, M. \& Ramasubramaniam, A. Two-dimensional material nanophotonics. Nat. Photon. 8, 899-907 (2014).

7. Mak, K. F., Lee, C., Hone, J., Shan, J. \& Heinz, T. F. Atomically thin $\mathrm{MoS}_{2}$ A new direct-gap semiconductor. Phys. Rev. Lett. 105, 136805 (2010).

8. Mak, K. F., He, K., Shan, J. \& Heinz, T. F. Control of valley polarization in monolayer $\mathrm{MoS}_{2}$ by optical helicity. Nat. Nanotechnol. 7, 494-498 (2012).

9. Zeng, H., Dai, J., Yao, W., Xiao, D. \& Cui, X. Valley polarization in $\mathrm{MoS}_{2}$ monolayers by optical pumping. Nat. Nanotechnol. 7, 490-493 (2012).

10. Bertolazzi, S., Brivio, J. \& Kis, A. Stretching and breaking of ultrathin $\mathrm{MoS}_{2}$. ACS Nano 5, 9703-9709 (2011).

11. Cooper, R. C. et al. Nonlinear elastic behavior of two-dimensional molybdenum disulfide. Phys. Rev. B 87, 035423 (2013).

12. Feng, J., Qian, X. F., Huang, C. W. \& Li, J. Strain-engineered artificial atom as a broad-spectrum solar energy funnel. Nat. Photon. 6, 866-872 (2012).

13. Radisavljevic, B., Radenovic, A., Brivio, J., Giacometti, V. \& Kis, A. Single-layer $\mathrm{MoS}_{2}$ transistors. Nat. Nanotechnol. 6, 147-150 (2011).

14. Lee, G.-H. et al. Flexible and transparent $\mathrm{MoS}_{2}$ field-effect transistors on hexagonal boron nitride-graphene heterostructures. ACS Nano 7, 7931-7936 (2013).

15. Fiori, G. et al. Electronics based on two-dimensional materials. Nat. Nanotechnol. 9, 768-779 (2014).

16. Wilson, J. A. \& Yoffe, A. D. Transition metal dichalcogenides discussion and interpretation of observed optical, electrical and structural properties. Adv. Phys. 18, 193-335 (1969).

17. Heising, J. \& Kanatzidis, M. G. Structure of restacked $\mathrm{MoS}_{2}$ and $\mathrm{WS}_{2}$ elucidated by electron crystallography. J. Am. Chem. Soc. 121, 638-643 (1999).

18. Eda, G. et al. Coherent atomic and electronic heterostructures of single-layer $\mathrm{MoS}_{2}$. ACS Nano 6, 7311-7317 (2012).

19. Duerloo, K. A. N., Li, Y. \& Reed, E. J. Structural phase transitions in two-dimensional Mo- and W-dichalcogenide monolayers. Nat. Commun. 5, 4214 (2014).

20. Keum, D. H. et al. Bandgap opening in few-layered monoclinic $\mathrm{MoTe}_{2}$. Nat. Phys. 11, 482-486 (2015).

21. Ali, M. N. et al. Large, non-saturating magne-toresistance in $\mathrm{WTe}_{2}$. Nature 514, 205-208 (2014)

22. Qian, X.-F., Liu, J.-W., Fu, L. \& Li, J. Quantum spin Hall effect in twodimensional transition metal dichalcogenides. Science 346, 1344-1347 (2014).

23. Park, J. C. et al. Phase-engineered synthesis of centimeter-scale $1 \mathrm{~T}^{\prime}$-and 2H-molybdenum ditelluride thin films. ACS Nano 9, 6548-6554 (2015).

24. Zhou, L. et al. Large-area synthesis of high-quality uniform few-layer $\mathrm{MoTe}_{2}$. J. Am. Chem. Soc. 137, 11892-11895 (2015).

25. Voiry, D. et al. Enhanced catalytic activity in strained chemically exfoliated $\mathrm{WS}_{2}$ nanosheets for hydrogen evolution. Nat. Mater. 12, 850-855 (2013).

26. Aizu, K. Possible species of ferroelastic crystals and of simultaneously ferroelectric and fer-roelastic crystals. J. Phys. Soc. Jpn 27, 387-396 (1969).

27. Salje, E. K. H. Ferroelastic materials. Annu. Rev. Mater. Res. 42, 265-283 (2012).

28. Salje, E. K. H. Phase Transitions in Ferroelastic and Co-Elastic Crystals (Cambridge Univ. Press, 1990). 
29. Kushima, A., Qian, X.-F., Zhao, P., Zhang, S.-L. \& Li, J. Ripplocations in van der Waals layers. Nano Lett. 15, 1302-1308 (2015).

30. Henkelman, G., Uberuaga, B. P. \& Jonsson, H. A climbing image nudged elastic band method for finding saddle points and minimum energy paths. J. Chem. Phys. 113, 9901-9904 (2000).

31. Sheppard, D., Xiao, P., Chemelewski, W., Johnson, D. D. \& Henkelman, G. A generalized solid-state nudged elastic band method. J. Chem. Phys. 136, 074103 (2012).

32. Tang, S., Mahanti, S. D. \& Kalia, R. K. Ferroelastic phase transition in two-dimensional molecular solids. Phys. Rev. Lett. 56, 484-487 (1986).

33. Huang, P. Y. et al. Grains and grain boundaries in single-layer graphene atomic patchwork quilts. Nature 469, 389-392 (2011).

34. van der Zande, A. M. et al. Grains and grain boundaries in highly crystalline monolayer molybdenum disulphide. Nat. Mater. 12, 554-561 (2013).

35. Cho, S. et al. Phase patterning for ohmic homojunction contact in $\mathrm{MoTe}_{2}$ Science 349, 625-628 (2015)

36. Brown, B. E. The crystal structures of $\mathrm{WTe}_{2}$ and high-temperature $\mathrm{MoTe}_{2}$. Acta Crystallogr. 20, 268-274 (1966).

37. Voiry, D., Mohite, A. \& Chhowalla, M. Phase engineering of transition metal dichalco-genides. Chem. Soc. Rev. 44, 2702-2712 (2015).

38. Chhowalla, M., Voiry, D., Yang, J., Shin, H. S. \& Loh, K. P. Phase-engineered transition-metal dichalcogenides for energy and electronics. MRS Bull. 40, 585-591 (2015).

39. Gordon, R. A., Yang, D., Crozier, E. D., Jiang, D. T. \& Frindt, R. F. Structures of exfoliated single layers of $\mathrm{WS}_{2}, \mathrm{MoS}_{2}$, and $\mathrm{MoSe}_{2}$ in aqueous suspension. Phys. Rev. B 65, 125407 (2002).

40. Enyashin, A. N. et al. New route for stabilization of $1 \mathrm{~T}-\mathrm{WS}_{2}$ and $\mathrm{MoS}_{2}$ phases. J. Phys. Chem. C 115, 24586-24591 (2011).

41. Bhattacharya, K. Microstructure of Martensite: Why It Forms and How IT Gives Rise to the Shape-Memory Effect (Oxford Univ. Press, 2003).

42. Salje, E. \& Zhang, H. Domain boundary engineering. Phase Transit. 82, 452-469 (2009).

43. Lebegue, S., Bjorkman, T., Klintenberg, M., Nieminen, R. M. \& Eriksson, O. Two-dimensional materials from data filtering and $\mathrm{Ab}$ Initio calculations. Phys. Rev. X 3, 031002 (2013)

44. Lin, Y.-C. et al. Single-layer $\operatorname{ReS}_{2}$ : two-dimensional semiconductor with tunable in-plane anisotropy. ACS Nano 9, 11249-11257 (2015).

45. Kresse, G. \& Furthmiiller, J. Efficiency of ab-initio total energy calculations for metals and semiconductors using a plane-wave basis set. Comput. Mater. Sci. 6, 15-50 (1996).

46. Kresse, G. \& Furthmiiller, J. Efficient iterative schemes for ab initio total-energy calculations using a plane-wave basis set. Phys. Rev. B 54, 11169-11186 (1996)
47. Blochl, P. E. Projector augmented-wave method. Phys. Rev. B 50, 17953-17979 (1994).

48. Perdew, J. P. et al. Atoms, molecules, solids, and surfaces: Applications of the generalized gradient approximation for exchange and correlation. Phys. Rev. B 46, 6671-6687 (1992).

49. Perdew, J. P., Burke, K. \& Ernzerhof, M. Generalized gradient approximation made simple. Phys. Rev. Lett. 77, 3865-3868 (1996).

50. Monkhorst, H. J. \& Pack, J. D. Special points for Brillouin-zone integrations. Phys. Rev. B 13, 5188-5192 (1976).

\section{Acknowledgements}

This work was primarily supported by the Center for Excitonics, an Energy Frontier Research Center funded by the US Department of Energy, Office of Science, Basic Energy Sciences under award no. DE-SC0001088. Our work was also supported by National

Science Foundation under grant no. DMR-1410636. Computational time on the Extreme Science and Engineering Discovery Environment (XSEDE) under the grant no.

TG-DMR130038 is gratefully acknowledged.

\section{Author contributions}

J.L. and W.L. designed the research. W.L. carried out the first-principles calculations W.L. and J.L. wrote the paper.

\section{Additional information}

Supplementary Information accompanies this paper at http://www.nature.com/ naturecommunications

Competing financial interests: The authors declare no competing financial interests.

Reprints and permission information is available online at http://npg.nature.com/ reprintsandpermissions/

How to cite this article: Li, W. et al. Ferroelasticity and domain physics in two-dimensional ransition metal dichalcogenide monolayers. Nat. Commun. 7:10843 doi: $10.1038 /$ ncomms10843 (2016).

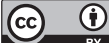

This work is licensed under a Creative Commons Attribution 4.0 International License. The images or other third party material in this article are included in the article's Creative Commons license, unless indicated otherwise in the credit line; if the material is not included under the Creative Commons license, users will need to obtain permission from the license holder to reproduce the material To view a copy of this license, visit http://creativecommons.org/licenses/by/4.0/ 